\title{
Megaciudades: contexto, cambio climático y el reto de la sostenibilidad urbana
}

\author{
Megacities: context, climate change and the challenge \\ of urban sustainability \\ Megacidades: contexto, mudanças climáticas e o desafio \\ da sustentabilidade urbana
}

\author{
Alejandro Mendoza Jaramillo. \\ M.Sc. Planificación Urbana y Regional por la Universidad de Buenos Aires. \\ Universidad Nacional de Colombia, Facultad de Artes. \\ Instituto Distrital de Patrimonio Cultural de la ciudad de Bogotá D.C. \\ almeja05@gmail.com \\ (iD) http://orcid.org/0000-0001-7332-4100
}

Recibido: febrero 19 de 2020

Aceptado: mayo 18 de 2020

Publicado: mayo 22 de 2020

\section{RESUMEN}

El presente artículo ofrece una breve revisión teórica de tres posturas académicas y políticas para hacer intervención en las ciudades, a la luz de las necesidades y retos que platean el Cambio Climático y la Sostenibilidad Urbana: Contención Urbana, Creación de Espacios y Acupuntura Urbana. De esta manera, interesa conocer los alcances, limitaciones, convergencias y divergencias de tales posturas, para generar debate frente a las necesidades actuales que muestra la planificación de las Megaciudades. El artículo finaliza con algunas reflexiones en torno a los retos que implica el Cambio Climático y la Sostenibilidad Urbana.

Palabras claves: Megaciudades, Sostenibilidad, Cambio climático, Ordenamiento Territorial.

\section{ABSTRACT}

This article offers a brief theoretical review of three academic and political positions to intervene in cities, in light of the needs and challenges posed by Climate Change and Urban Sustainability: Urban Containment, Creation of Spaces and Urban Acupuncture. In this way, it is interesting to know the scope, limitations, convergences and divergences of such positions, to generate debate against the current needs that the planning of Megacities shows. The article ends with some reflections on the challenges of Climate Change and Urban Sustainability.

Key words: Megacities, Sustainability, Climate change, Territorial Planning. 


\section{RESUMO}

Este artigo oferece uma breve revisão teórica de três posições acadêmicas e políticas para intervir nas cidades, tendo em vista as necessidades e os desafios impostos pelas mudanças climáticas e sustentabilidade urbana: contenção urbana, criação de espaços e acupuntura urbana. Dessa forma, é interessante conhecer o alcance, as limitações, as convergências e as divergências de tais posições, para gerar um debate diante das necessidades atuais que o planejamento das Megacidades mostra. $\mathrm{O}$ artigo termina com algumas reflexões sobre os desafios das mudanças climáticas e da sustentabilidade urbana.

Palavras-chave: Megacidades, Sustentabilidade, Mudanças Climáticas, Planejamento Territorial.

\section{INTRODUCCIÓN}

El año 2007 se registró como histórico, pues, a partir de entonces, la mitad de la población mundial vivía en ciudades; "el mundo alcanzará un hito invisible pero trascendental: por primera vez, más de la mitad de su población humana" (UNFPA, 2007, p.1). Esto no es casual, pues, aunque aquí no interesa agotar la discusión al respecto, es pertinente destacar tres causas que permiten explicar este fenómeno: los cambios tecnológicos, que apalancaron nuevos estilos de vida; el reforzamiento de Modelos Económicos y de Ordenamiento Territorial desequilibrados, que le restan oportunidades a las comunidades rurales,; así como en el caso de los países menos desarrollados, se mantienen unas tasas de crecimiento vegetativo elevadas que, en parte, explican por qué continúan Ilegando contingentes migratorios de población rural a las ciudades.

Un primer factor que se considera clave, para entender el desarrollo extensivo de las ciudades, son los cambios tecnológicos, que apalancaron nuevos estilos de vida. Así, pues, el desarrollo del transporte terrestre, con el uso de ferrocarriles en un primer momento y de vías pavimentadas posteriormente, eliminó las fronteras y mejoró la posibilidad de elegir nuevas localizaciones, independientemente de la proximidad. (Ainstein, 2012; Buzai, 2006; Angel, 2015). Su surgimiento e intensificación aparecen como resultado del enraizamiento de profundos cambios en las formas de construir el espacio; de la marcada desigualdad en la conectividad y accesibilidad a los equipamientos urbanos; así como de renovados y diferenciados procesos de socialización y estilos de vida que dieron énfasis a la expansión urbana basada en modelos tan opuestos como lo son la oferta residencial cerrada de baja densidad, de un lado, y las urbanizaciones de la informalidad/ ilegalidad, del otro.

En cuanto al reforzamiento de Modelos Económicos y de Ordenamiento Territorial desequilibrados, aunque son muchas las razones que explican este fenómeno, aquí cabe destacarse que la inserción de las ciudades en los mercados mundiales, en el contexto de la globalización económica, hizo que algunas urbes se convirtieran en una suma fragmentaria, separada o compuesta de nudos o puntos específicos (Mendoza, 2015), en los cuales invertir para generar grandes centros de comunicación, producción y comercio, en detrimento de las ciudades pequeñas e intermedias: transfirió a las ciudades algunas funciones básicas del desarrollo económico y social que anteriormente ejercían los países, por lo que estas debieron empezar a ser competitivas -lo que implica ser eficientes $\mathrm{y}$ atractivas para la gente- $\mathrm{y}$ mejorar sus condiciones de vida. Por ello, se convirtieron en los centros de poder desde los que se impulsaban las medidas tendientes a que su inserción eficiente en el nuevo orden económico internacional fuera efectiva (Mendoza, 2014).

Ainstein (2012), ONU-HABITAT (2016), Sorensen y Okata (2011) señalan que el crecimiento urbano de las ciudades ha sido caótico y desbocado, aun con los esfuerzos que los Gobiernos locales han hecho para controlar la localización de ciertos 
usos del suelo y gestionar de manera significativa la producción del territorio. En la fuerza de atracción que ejerce cada ciudad sobre las áreas circundantes, entran en juego muchos factores particulares, no obstante, todas las ciudades, independientemente de su escala, han tendido a mostrar el mismo patrón de atracción, buscando posicionarse en una centralidad urbana, según sus recursos y jerarquía.

\begin{abstract}
Algunos investigadores han elaborado diferentes modelos para explicar las dinámicas que definen la estructura urbana actual de las ciudades: anillos concéntricos de Burgess; de sectores de Hoyt y de anillos múltiples de Harris y su aplicación en Latinoamérica con Borsdorf (2003), Janoschka (2002) y Jaramillo (1993, 1999)'. Autores como Abba (2010), Lorrain (2011) y Pírez (2010) han abordado la temática desde la perspectiva de la complejidad que supone el continuo urbano que incluye múltiples territorios en la formación de una gestión urbanística interjurisdiccional, en tanto que, salvo contadas excepciones, no se llegan a instancias de concertación que desemboquen en una gestión urbanística de escala supralocal (v.gr. metropolitana).
\end{abstract}

Porúltimo, en cuanto al crecimiento poblacional y los contingentes migratorios, es interesante llamar la atención frente al hecho de que este fenómeno se presenta de manera diferencial, según el desarrollo socioeconómico del país en cuestión, pues, mientras en América del Norte, Japón y Europa se estabilizan y tienden a la baja los indicadores demográficos, $y$, por lo tanto, se puede suponer que también se enlentece su crecimiento urbano, en Latinoamérica, África y Asia, el crecimiento de la cantidad de habitantes urbanos, y su casi inevitable expansión urbana, estará caracterizada por la informalidad (Jayan, 2009, Sorensen y Okata, 2011 y Ángel, 2015). Todo esto toma tal dinámica que, de acuerdo con la Organización de Naciones Unidas (ONU),

1. Algunos fenómenos que resultan comunes en los autores son: importancia que tuvieron las migraciones rural-urbano y los fuertes cambios demográficos, la inadecuación de los usos del suelo con la producción de tipo tradicional, la primacía de la ciudad capital frente al resto urbano (centro altamente especializado y macrocefalia), el acelerado crecimiento de la mancha urbana con intensiva fragmentación y declive de la densidad/ compacidad, el elevado número de personas en situación de vulnerabilidad social, además de la fuerte marginalidad física y segregación intra urbana. para el año 2015, la población mundial que vivía en ese tipo de asentamientos superaba el billón de personas. Esta cifra plantea serios interrogantes frente a la Sostenibilidad de las ciudades en el corto y mediano plazo.

Los asentamientos irregulares e informales, presentes en todas las Megaciudades de los países en desarrollo, fueron considerados, en principio, como manifestaciones negativas o cánceres del sistema urbano, aunque la perspectiva analítica cambió al detectarse que eran más frecuentes de lo esperado. En ese sentido, se encontró, entonces, que los residentes de tales áreas tomaron la única posibilidad que encontraron para acceder al suelo urbano y proveerse de una vivienda. Se podría establecer como un "círculo virtuoso": las personas llegan a la ciudad, la economía del lugar se dinamiza con la llegada de esta población, en tanto que representan mano de obra para distintas labores (principalmente no calificada), así como nuevos consumidores de bienes y servicios. Las necesidades habitacionales llevan a que estos nuevos habitantes urbanos ocupen barriadas improvisadas que, con el paso del tiempo, se consolidarán y, eventualmente, serán legalizadas por los respectivos Gobiernos locales ${ }^{2}$

\footnotetext{
2. Desde la perspectiva de las viviendas, en el mercado formal los costos de aquellas unidades habitacionales que atienden adecuadamente la producción de ciudad a nivel local (con andenes, vías, alumbrado público, parques locales) están contenidos en el precio de la vivienda, es decir, se trasladan al comprador. Las obras y los costos se denominan cargas locales, por lo que el comprador está dispuesto a pagarlas. Por su parte, el mecanismo paralelo con el que la población pobre es atendida para adquirir una vivienda es el mercado informal: el urbanizador ilegal o "pirata" adquiere tierra con anticipación en la zona clasificada por las normas como rural, a precios muy bajos, elude todo el proceso de aprobación y de manera furtiva vende lotes sin urbanizar. Marca unos linderos para lotes, dejando espacios reducidos para las vías, pues el negocio es sacar la mayor cantidad de lotes; no cuenta con servicios públicos domiciliarios (ni siquiera previstos por las correspondientes empresas), no existen los equipamientos urbanos ni los sistemas de alumbrado público, alcantarillado ni calles, etc. Estos "lotes sin servicios" se venden a precios accesibles para su nicho de mercado, consiguiendo de paso ganancias entre 10 y 20 veces el valor que pagó por ellos. Comienza para el comprador un proceso de capitalización lenta, en la cual construye su casa por partes e inicia la gesta para la consolidación y legalización del barrio. Asistido por el Estado, al cabo de los años adquiere buena parte de la condiciones urbanas que se esperaba hiciera el urbanizador formal y pagara el consumidor de vivienda, y decide legalizar. El costo que resulta es enorme, casi el mismo que si se hubiera urbanizado formalmente desde un inicio con el agregado de que el barrio creado por la informalidad queda en condiciones precarias de espacio público y de equipamientos. Son muchas las variables que intervienen y determinan el cómo se presenta la dinámica de expansión urbana informal, no obstante, en el presente artículo se presenta este modelo simplificado a manera de ejemplo.
} 
Aquí es válido señalar que la relación de los residentes informales con las actividades económicas urbanas conlleva a unas condiciones críticas: consiguen los peores trabajos con alto riesgo, dificultad, bajos pagos y/o en la informalidad, sus hijos difícilmente logran ingresar al sistema educativo o ser atendidos por los servicios de salud. Además, es común asociar el desarrollo informal con altas densidades de población, la ineficiente prestación de los servicios de transporte público, alta polución ambiental, exposición a las peores condiciones ambientales (desastres naturales), inadecuados sistemas de acueducto y alcantarillado, a lo que se suma la ausencia de políticas públicas que aborden orgánicamente el déficit cualitativo y cuantitativo de viviendas. Al respecto, Sáenz (2006) retoma algunos elementos descritos por el Informe de Asentamientos Humanos que realizó la ONU en 1996, para señalar las características comunes de los hogares con déficit en Latinoamérica, resaltando la correspondencia existente entre la ocupación e ingresos de la población y el déficit de vivienda.

Respecto al marco político-institucional, la informalidad no solo tiene impactos económicos y urbanos, sino que tiene altos costos políticos, dado que algunos gobernantes de turno se aprovechan de la precaria situación de su población para conseguir votos, proclamándose como defensores del derecho a la ciudad de los menos favorecidos.

Así, la informalidad de las ciudades se presenta como un fenómeno imposible de detener, cuyo conflicto urbano no se puede resolver porque la norma prohíbe dotar de bienes y servicios a los barrios ilegales, pero, al tiempo, tampoco se puede aplicar la ley a la fuerza, por lo que la legalización se volvió el mecanismo de resolución del conflicto. No obstante, los barrios legalizados sin consolidar se convierten en los principales generadores de presión sobre los presupuestos locales, debido a las necesidades de infraestructura (construcción de vías, pavimentación, construcción de acueductos, alumbrados) y de los presupuestos de las empresas de servicios públicos.

Sendas dinámicas fueron abordadas desde diferentes perspectivas por académicos y técnicos, los cuales formularon y desplegaron una gestión urbanística con diversidad de instrumentos de intervención territorial, de acuerdo con los recursos propios. En ocasiones, se formó una contradicción entre un proceso territorial con implicancias metropolitanas y regionales, la expansión urbana descontrolada, y la profusión de instrumentos urbanísticos locales desde los Gobiernos municipales, los cuales resultaron en normativas fragmentarias que buscaron regular, viabilizar o legalizar aspectos parciales, sin comprender el fenómeno como un todo.

Desde diferentes Gobiernos locales se implementaron políticas y teorías urbanísticas de distinto tipo, cuya formulación y resultados dependió de los recursos con que se contaba (de tipo económico, institucional, tecnológico y social), sino que principalmente estaban determinados por el pensamiento académico y político del momento de su formulación e implementación. En este sentido, el objetivo del presente artículo es ofrecer una breve revisión teórica de tres posturas académicas y políticas, para hacer intervención en las ciudades a la luz de las necesidades y retos que platean el Cambio Climático y la Sostenibilidad Urbana: Contención Urbana, Creación de Espacios y Acupuntura Urbana. Aquí se considera que las transformaciones urbanas ocurridas en contextos recientes, deben hacer repensar los paradigmas con los cuales nos acercamos a la ciudad, para lo cual es necesario evaluar las posturas existentes y proponer formas aterrizadas, pero novedosas, de intervención. En este sentido, se presentan estas posturas, con el fin de generar debate frente a sus alcances, limitaciones, convergencias y divergencias.

Cabe señalar que en el presente documento se mencionan algunas de las principales 
problemáticas derivadas de las grandes ciudades $^{3}$, pero que no se profundiza en ellas, pues el énfasis está puesto en las posturas de intervención en la ciudad; además, dada la variedad de situaciones que presenta cada una de las Megaciudades, aquí interesó revisar las características comunes que resultaran de interés para los técnicos y profesionales de las tres posturas académico-técnicas elegidas para el análisis comparativo.

Ahora bien, el orden del texto es el siguiente: en el segundo apartado se presenta el contexto actual de las Megaciudades, su incidencia en el Cambio Climático y el reto que significan para la Sostenibilidad, para luego entrar en el tercer apartado a las tres posturas de intervención señaladas. El documento finaliza con algunas reflexiones frente a lo descrito, a manera de un Ilamado a repensar la lógica con la que se abordan las urbes en los instrumentos de ordenamiento territorial actuales.

\section{Megaciudades: Contexto, Cambio Climático y el Reto de la Sostenibilidad Mundial}

Por Megaciudades se entiende las aglomeraciones urbanas que albergan a diez millones de personas, según la definición establecida por la ONU y según datos publicados en el último informe sobre el Estado de las Ciudades del Mundo. Para el año 2015, alcanzaban un número de 29 en el mundo, más 44 ciudades grandes, en las que vivían cerca de 700 millones de personas o aproximadamente el $10 \%$ de la población urbana mundial. La mayoría de ellas, el $80 \%$, están localizadas en países en vía de desarrollo de África, Asia o América Latina. Algunos autores señalan que el crecimiento de las Megaciudades de países desarrollados como Tokio y New York se ralentiza, contrario a lo que ocurre en países en vías de desarrollo como Ciudad de México en México, Lagos en Nigeria, Sao Paulo en Brasil o Bangkok en Tailandia.

3. Para consultar los casos de estudio se recomienda ver los siguientes autores: Ainstein, 2012; Angel, 2015; BID, 2014; Kraas, Aggarwal, Coy y Mertins, 2014; Jayan, 2009; Lorrain, 2011; Sorensen y Okata, 2011.
La consolidación de Megaciudades en distintos rincones del planeta, transforma el mapa geoestratégico del mundo, con ciudades de mayor población y que producen el PIB anual de muchos países, alcanzando el $70 \%$ del crecimiento económico nacional de Francia y Japón, haciendo pensar en la necesidad de superar la planificación anclada al territorio, para concebirlo como sistemas estratégicos de Megaciudades interconectadas; a la vez que demuestra que, inevitablemente, las urbes van a tener un poder político muy grande en un futuro cercano (Sustentabilidad Responsable, 2016; El País, 2016; Khanna, 2016).

Esta idea se refuerza si tenemos en cuenta que tales urbes constituyen los puntos focales de la globalización, por lo que albergan un amplio espectro de habilidades, multiculturalidad y potencial humano por aprovechar:

"Las Megaciudades son espacios de atracción, de relación de multiculturalidad y también de contradicción. Todos los mundos están reunidos en ellas: las oficinas de las multinacionales, que compiten en edificios emblemáticos; los barrios residenciales de lujo; las viviendas de los asalariados, que repiten modelos arquitectónicos, creando banalidad urbanística; y la pobreza más absoluta, el chabolismo más degradado" (El País, 2008).

El auge económico de una Megaciudad implica fuertes presiones para su extensión urbana, "las Megaciudades crecen más rápido que su infraestructura. Este crecimiento urbano desbocado puede fomentar altos volúmenes de tráfico, altas concentraciones de producción industrial, sobrecarga ecológica, mercados de tierras y propiedades no regulados y dispares, desarrollo de viviendas insuficiente $y$, en algunos casos, tales extremos de pobreza y riqueza viviendo al lado" (Jayan, 2009, p.5)4 De esta manera, Jayan (2009) y Jorge Olcina

\footnotetext{
4. "Megacities grow faster than their infrastructure. This run-away urban growth can foster high traffic volumes, high concentrations of industrial production, ecological overload, unregulated and disparate land and property markets, insufficient housing development and, in some cases, such extremes of poverty and wealth living side by side".
} 
(2008) llaman la atención sobre la forma como se aborda la Megaciudad: su comprensión debe hacerse considerando las dinámicas ampliadas que generan, la nueva complejidad que invisten en aspectos como lo físico, social y económico, por ser sede de complejas interacciones entre procesos demográficos, sociales, políticos, económicos y ecológicos. Su importancia radica, precisamente, en que sus transformaciones impactan al resto del mundo, pues sus efectos superan las fronteras administrativas locales y toman significancia regional, nacional y global.

Ahora bien, distintos autores académicos e institucionales coinciden en señalar que las Megaciudades del mundo están generando serios problemas en la calidad del ambiente mundial y que tienen incidencia directa sobre el calentamiento global $^{5}$, tan vigente en nuestros días, al tiempo que preocupa su Sostenibilidad $^{6}$ en el tiempo. En ese sentido, estas urbes con sus altas demandas de bienes y servicios constituyen en un enorme desafío para las administraciones públicas. De acuerdo con la literatura especializada, son diversos los retos a la gestión local que plantean el Cambio Climático y la Sostenibilidad Urbana. Estos son:

a. Crecimiento Intensivo de la Población: Incide en que la infraestructura urbana sea insuficiente, demanda creciente de bienes y servicios y una desigualdad económica que lleva a la marginalidad con todo lo que implica (hacinamiento, aumento de la criminalidad, las enfermedades, el narcotráfico y la inseguridad);

\footnotetext{
5. Por este concepto se entiende en general el aumento en la temperatura media de la tierra y sus respectivos impactos en fenómenos como el calentamiento de océanos, derretimiento de polos y aumento de la temperatura global. En este se presentan temperaturas extremas, olas de calor y fríos, aumento de las zonas afectadas por las sequías e intensificación de eventos como los ciclones tropicales (Hansjürgens y Heinrichs, 2014). De acuerdo con algunos autores, la intervención del hombre en los últimos siglos intensificó el cambio climático y su explicación más consistente se encuentre en la concentración alta de los gases de efecto invernadero en la capa de ozono.

6. Entendida desde la Declaración de Río de 1992 como "las necesidades del presente sin comprometer la capacidad de las futuras generaciones para satisfacer las propias", es una temática que ha tomado fuerza desde 1972, fecha en que se realizó la Conferencia de las Naciones Unidas sobre el Medio Ambiente en Estocolmo.
}

b. Presiones para la Ocupación de Suelos: Para distintos usos y necesidades crecientes de vivienda -que en algunos casos significa desperdicios por la expansión fragmentada;

c. Limitaciones de las Administraciones Locales: Dependencia de los Gobiernos estatales que limitan la independencia de las administraciones locales, además de otro tipo limitaciones administrativas;

d. Parque Automotor: Dificultades en el funcionamiento urbano ocasionadas por el aumento del parque automotor (creciente tráfico, congestión y viajes vivienda-trabajo), lo cual se refuerza con deficientes sistemas de transporte público;

e. Extensión de la Mancha Urbana: La extensión descontrolada de la mancha urbana apoyada en la proliferación de barrios pobres y de suburbanización de baja densidad;

f. Contaminación de Recursos Naturales: La intensa demanda y Contaminación de los Recursos Naturales necesarios para la subsistencia humana (principalmente aire, agua y suelo), con el consecuente deterioro de la salud pública ${ }^{7}$ y la devastación de ecosistemas naturales (alto consumo de recursos derivado de la gran demanda eléctrica y de agua de los habitantes). A esto se une el hecho de que las Megaciudades son productoras de residuos y desechos de todo tipo, que no siempre son bien gestionados;

\section{g. Dependencia del Petróleo: Alta dependencia a una fuente de energía altamente contaminante e ineficiente en rendimiento (el petróleo), haciendo que en las Megaciudades se produzcan y concentren altas tasas de gases de efecto invernadero. La ONU apunta que "el desarrollo de las ciudades más importantes}

\footnotetext{
7. De acuerdo con Jayan (2009) se estima que el impacto económico de la contaminación en áreas urbanas en general es muy alto, en términos de pérdida de productividad y los costos de salud: de acuerdo con un informe del Banco Asiático de Desarrollo (BAD), en 2002 se estimó que en muchos países superará el $10 \%$ del Producto Interno Bruto (PIB); a lo que se suma la proliferación de enfermedades, altas tasas de mortalidad y las cepas resistentes a los fármacos dadas las condiciones ambientales letales.
} 
del planeta no puede ser infinito. Son enormes sumideros de recursos hídricos, energéticos y de materiales de construcción" (El País, 2008);

h. Pérdida de Espacios Públicos: Paulatina pérdida de espacios públicos de socialización junto con la consolidación de nuevos escenarios privados;

i. Deterioro de la Infraestructura Instalada: La escasez de capital agrava aspectos como el deterioro de la infraestructura instalada, entre otros;

j. Preparación para la Ocurrencia de Desastres Naturales Pronosticados como consecuencia del Cambio Climático (impacto sobre los ecosistemas y aprovisionamiento de servicios, aumento de las temperaturas, precipitaciones, del nivel del mar y de episodios climáticos extremos, con los consecuentes costos económicos) a través de Estrategias de Mitigación y/o Adaptación: En general, estas urbes representan focos de riesgo global, en tanto que su densidad aumenta la vulnerabilidad de las personas a peligros naturales y artificiales en el marco del Cambio Climático (zonas sensibles al clima como costas, áreas propensas a inundación o zonas que sufren de escasez de agua y sequías), al tiempo que ella misma es la productora de tales condiciones (Jayan, 2009; Hansjürgens y Heinrichs, 2014; BID, 2014).

\section{Propuestas de Intervención Urbana}

Ahora bien, con el ánimo de recoger algunas propuestas de intervención que se pueden encontrar en la literatura especializada, en el presente artículo se seleccionaron tres alternativas analíticas que varían en forma y contenido para abordar lo arriba descrito: una primera postura, que podríamos llamar más "tradicional" y que en este artículo denominamos "Paradigma de Contención Urbana"; una segunda, más integral y que propone soluciones ajustables a los distintos contextos de las Megaciudades Ilamada aquí como "Paradigma de la Creación de Espacios"; y una última alternativa, más enfocada en el proyecto urbano, conocida como "Acupuntura Urbana". Antes de entrar en la descripción de cada una de estas tres corrientes, cabe señalarse que se utilizan tales categorías para agrupar las distintas posturas que se pueden encontrar en la gestión local, aunque en la presente investigación no se espera agotar la discusión que existe al respecto, ni se señala alguna postura como la correcta, pues es un tema abierto, vigente y que necesita de la reflexión y aporte de profesionales de todas las ciencias; por lo tanto, en la literatura especializada se pueden encontrar otras ideas y propuestas a las aquí presentadas.

\section{Paradigma de la Contención Urbana}

Siguiendo a Ángel (2015), en este paradigma se encuentran todos los trabajos académicos, técnicos y políticos que, frente a la expansión de la ciudad, proponen una suerte de medidas de contención universales, con las cuales desincentivar las dinámicas en ciernes. Si bien no existe un consenso sobre las principales características que se podrían alinear a este paradigma, sí se encuentra en académicos y técnicos expertos un discurso común que rechaza el crecimiento urbano y lo desglosa en los siguientes aspectos: instrumentos de ordenamiento del suelo que propenden por la densificación del área construida de la ciudad y disminuir la fragmentación en los bordes, tales como límites perimetrales relativamente estrictos; preocupación por el crecimiento poblacional, sobre todo cuando se hacen comparaciones de las tasas de crecimiento de las grandes ciudades con las pequeñas; preocupación por la expansión descontrolada de los asentamientos informales; énfasis en la incidencia ambiental que tiene la ciudad cuando se proclama la Sostenibilidad como fin que justifica muchos medios (si no todos).

En ese mismo sentido, se considera la expansión urbana como un mal que puede evitarse por distintos mecanismos: la fijación de límites urbanos estrictos, controles de 
migración nacionales e internacionales; declaratoria de extensas zonas que rodean la ciudad como espacios de interés o cinturones verdes, o, al contrario, la densificación y reutilización de franjas de suelo en desuso/ subuso de áreas consolidadas de la ciudad, aplicando mayores factores de ocupación del suelo y proyectos de revitalización.

La intervención del Estado en sus territorios es urgente, pues es el principal responsable de corregir los escenarios tendenciales por unos ideales de Sostenibilidad que pone el foco en el factor ambiental. A su vez, se destaca el rol importante que deben jugar la Tecnología y la Infraestructura en la eficiencia mejorada de esas ciudades densificadas: sistemas de transportes públicos óptimos; parques públicos articulados; una institucionalidad actualizada y eficiente, que articule enfoques participativos con las comunidades locales y reformule las finanzas urbanas disponibles; ampliación de vías y servicios públicos/ saneamiento asegurado para toda la población urbana con un óptimo manejo de los residuos sólidos.

Esta postura, si bien es sincera en sus intenciones de frenar el caos urbano, deja de lado importantes consideraciones que determinan su éxito: los Gobiernos locales de muchas Megaciudades de los países en desarrollo no cuentan con los recursos económicos suficientes que son necesarios para hacer las intervenciones propuestas, pues una intervención en suelo altamente densificado, como el de los asentamientos informales, genera altas erogaciones que terminan desdibujando el objetivo inicial. De hecho, es bien conocido que toda intromisión por parte de un Gobierno genera presiones y especulaciones en los propietarios y habitantes del sector a intervenir, lo que produce efectos contrarios a los esperados. A su vez, las medidas estrictas de control a la expansión urbana han mostrado no ser eficaces en ninguna de las modalidades que se han aplicado, además que proponer una densificación urbana en alturas, puede significar aumentar los riesgos ambientales y vulnerabilidad a los que está expuesta la población, al tiempo que, si se aplica de forma descuidada, representaría una disminución en la calidad de vida de la población.

En un sentido amplio, las propuestas de este paradigma pecan por dos razones: de un lado, se considera a la ciudad o Megaciudad como un ente aislado sobre el que hay que regular ciertas actividades para obtener los resultados esperados, sin tomar en cuenta las dinámicas regionales, nacionales e internacionales; de hecho tal vez en ese contexto más amplio se podrían encontrar algunas respuestas a las preguntas sobre cómo intervenir en la ciudad de interés; $y$, del otro, se considera que las recetas exitosas se pueden aplicar de igual forma en otras ciudades con el mismo problema, sin considerar las particularidades de cada urbe.

Son distintos los ejemplos de ciudades que han aplicado algunos o todos los principios de este paradigma: Perímetro de Crecimiento Urbano de Portland en Estados Unidos, Restricción a la Movilidad en China, Desalojo de Ciudades para Retornar la Población a las Áreas Rurales en Camboya, o, en nuestro país, la Definición de un Perímetro Urbano para Bogotá D.C.

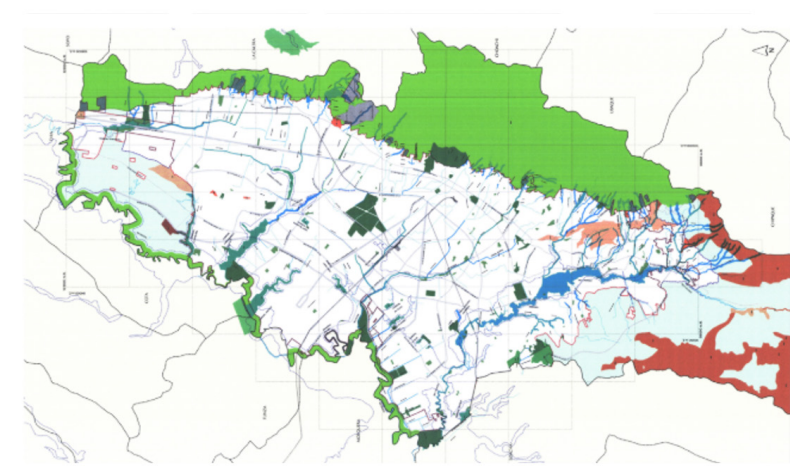

Figura 1. Plano de la Estructura Ecológica Principal de la Ciudad de Bogotá D.C.

Fuente: Secretaría Distrital de Planeación, SDP (2019). 


\section{Paradigma de la Creación de Espacios}

Ángel (2015) sintetiza y agrupa este paradigma basado en un principio: hacer los preparativos necesarios para acomodar la expansión de la ciudad; para lo cual formula cuatro pilares: Expansión Inevitable, Densidades Sostenibles, Viviendas Dignas y Obras Públicas. Esta postura académica es diametralmente opuesta a la anterior, pues considera que la expansión de las ciudades se puede acompañar de manera sostenible partiendo de la premisa de que su contención es imposible, por lo que es necesario asegurar unas condiciones mínimas que permitan mantener la buena calidad de vida e integrando a todas las personas que llegan a la urbe.

El paradigma postula la necesidad de proyección de suelos necesarios de acuerdo con las tendencias de población actual y considerando algunos aspectos técnicos específicos, como la reserva para vías y parques públicos. Ángel (2015) sostiene, además, basado en estadísticas, que, contrario a lo que el sentido común indica, se debe desescalar la preocupación por el crecimiento poblacional de las grandes urbes, pues, aunque distintos académicos han intentado calcularlo, aún no existe un tamaño de población que sea eficiente, así como evidencia que las Megaciudades no están creciendo en un ritmo más acelerado que las ciudades de otras escalas, pues todas mantienen una tasa relativa de crecimiento natural. Así, afirma que en el caso de los países subdesarrollados, sus metrópolis seguirán en expansión y es por ello que propone una alternativa sostenible para la planificación de esos territorios.

Un primer aspecto que aborda Ángel (2015) es que en los países que cuentan con Megaciudades son estructuras de gobiernos débiles, que no cuentan con los recursos económicos necesarios para su función, por lo que es menester reforzar la gestión posible considerando los contextos diferentes, con configuraciones, necesidades, retos y oportunidades específicas.
En relación con aspectos que se han identificado como "claves", por su interrelación con el cambio climático, como la creciente tendencia a la suburbanización de baja densidad y el uso del automóvil particular, se debe evaluar el panorama actual que ha llevado a que la ciudad no ofrezca buenas alternativas de movilidad, al tiempo que se debe incidir sobre la expansión espontánea informal, lo cual redundaría en la reducción de la vulnerabilidad de esos habitantes y la disminución en los costos que se requerirían para llevar a cabo una posterior intervención.

Para finalizar, en el mismo paradigma se encuentra un análisis de las transformaciones de la ciudad, según su expansión física y transformación relaciona, tendiendo a diseñar en el marco de la economía globalizada, ciudades policéntricas. Esta es la fase en la cual se encuentran la mayoría de Megaciudades del mundo, y no por ello es una característica negativa, pues, por el contrario, presenta la consolidación de subcentros especializados en todas las periferias metropolitanas, lo cual reduce los tiempos de transporte de los trabajadores, llegando inclusive a situaciones en las que no necesitan ir a la ciudad central, lo cual redunda en una reducción en la energía consumida y en la consolidación de las tramas urbanas de esas zonas.

Ahora bien, al igual que el anterior paradigma, en el de Creación de Espacio se considera como necesaria la intervención de los Gobiernos locales pues es el único actor capaz de generar espacios dotados de infraestructura que posiblemente no son interesantes para la lógica económica del capital privado (como vías y transporte público). No obstante, se debe examinar las situaciones y formas adecuadas de intervenir, pues, en el caso de las viviendas, las regulaciones pueden generar más impactos negativos que los esperados, a la vez que se considera clave una sana articulación con el libre mercado (sector privado). 
Como contracara, en el paradigma se deja de lado algunas cuestiones que son importantes: los determinantes ambientales especiales que determinan la forma particular de la ciudad y que son evaluados de forma general, tal vez porque no se podría entrar en tanto detalle para las ciudades analizadas por Ángel (2015); al tiempo que se sobrevalora la posibilidad de aumentar la producción agrícola para brindar la seguridad alimentaria de los habitantes a través de nuevas técnicas tal vez transgénicas, como si esto dependiera exclusivamente de la política pública o del Gobierno local en turno. Algo parecido ocurre con el llamado a establecer buenos sistemas de transporte público, pues no logra especificar cuál actor sería el responsable de la prestación de tal servicio, si el sector público o el privado, y cuál sería el sistema más acorde para una prestación eficiente.

Resta señalar que en las ciudades ecuatorianas de Manta y Milagro, se utilizó este paradigma para la formulación de un plan de expansión urbana. Otros referentes y pioneros en su aplicación fueron el Plan de Ensanche elaborado por Cerdá para Barcelona en España y el Plan de 1811 de los Comisionados de la ciudad de New York en los Estados Unidos. En Bogotá D.C. se encuentran diseñados algunos planes parciales, aplicando los principios de este paradigma; uno de los más debatidos es conocido como la Operación Estratégica Nuevo Usme al sur de la capital.

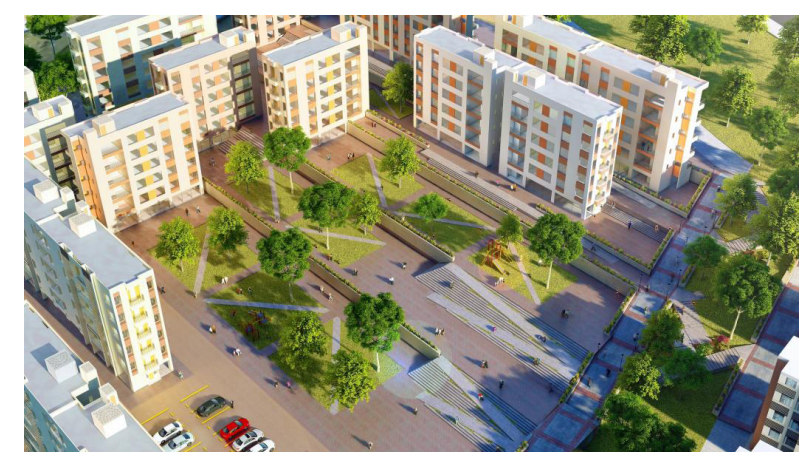

Figura 2. Render de la Operación Estratégica Nuevo Usme, Bogotá D.C.

Fuente: Página web Bogota.gov.co

\section{Acupuntura Urbana}

En esta propuesta se encuentra a Oriol Bohigas y Jaime Lerner (2005), los cuales, desde los planos político y académico, adoptaron una postura que integra algunos elementos de los paradigmas anteriores: la Acupuntura Urbana. En esta, se entiende que el éxito del planeamiento depende de las acciones puntuales que se realicen sobre las ciudades, para comenzar su transformación y lograr que tenga efecto sobre el resto del sistema urbano, orientado a buscar el

"equilibrio vital de las ciudades, basado en un modelo de ciudad en el que tiene prioridad la equidad, la convivencia y la cohesión social, el desarrollo sostenible, la habitabilidad, la solidaridad, la cultura y la educación urbana, al igual que la compacidad urbana, la conservación y la rehabilitación del patrimonio histórico y popular" (Tarchópulos, 2006, p. 136).

Así, se entiende que la Acupuntura se basa principalmente en la intervención sobre aspectos puntuales de la ciudad que se deseen estimular en simultáneo, para regenerarlos, principalmente espacio público. Se le da un peso importante a la intervención sobre el elemento vivienda, porque consideran que es la esencia de la ciudad, representa la optimización de recursos ecológicos y económicos, así como incidir directamente sobre las lógicas de crecimiento urbano.

Desde este enfoque, se considera menester optimizar los recursos económicos y ecológicos en la intervención urbana, en tanto que no en todos los países en los que se requiere hacer una intervención urbanística se cuenta con los recursos suficientes por su amplio coste, y que hablamos de contextos urbanos consolidados y densificados, por lo que se deben priorizar las obras y fomentar acciones que activen el desarrollo sostenible en sus urbes.

Esta postura ofrece una crítica directa al primer paradigma descrito en este artículo: 
"La zonificación de la ciudad planteado por el movimiento moderno resulta difícilmente aplicable en un urbanismo contemporáneo que busca y necesita la pluralidad del uso del suelo (...) El urbanismo moderno que actúa por medio de zonificaciones urbanas, queda por tanto obsoleto. Actualmente resulta sumamente costoso, insostenible 0 ficticio"*. (Barahona, 2008, p. 293).

En cambio, resulta consecuente incentivar y construir redes o sistemas de acupunturas urbanas entretejidas, para que actúen de manera complementaria. Estas redes se pueden adherir a los contextos urbanos existentes y equilibrar las necesidades de los ciudadanos, como el caso de la vivienda y el espacio público. Según los autores, la acupuntura asegura una intervención en la ciudad con secuelas en las regiones, al tiempo que se favorece la compactación (no como hacinamiento) y el equilibrio. Esto último favorece la densificación de los espacios, disminuyendo los desplazamientos cotidianos y representando beneficios, en términos energéticos y económicos.

La principal crítica que se le hace a esta postura es que la utilización de esta técnica, que apela a expropiaciones o desalojo, significa la ruptura de dinámicas sociales bien establecidas por el afán de reinsertar tales sectores en las dinámicas económicas del resto de la ciudad, es decir, generar valor en sectores subutilizados de la ciudad, a partir del desplazamiento de los pobladores originarios. En el mismo sentido, no se hace evidente en su argumentación, es que la elección de los sectores a intervenir corresponde principalmente a razones económicas, zonas de relevancia económica, más no a las necesidades reales que tenga la ciudad.

Por otra parte, se puede señalar que los beneficios que se pueden detectar de las intervenciones hechas -puntuales 0 en red- no necesariamente inciden en la ciudad como un todo, sino que refuerzan patrones de segregación urbana, con sectores altamente intervenidos y cuidados, y otros que evidencian la desatención por parte de los Gobiernos de turno. Además, con esta forma de intervenir en la ciudad, no se da respuesta a todas las necesidades que tienen los habitantes de una ciudad; de hecho no se aproxima a responder carencias estructurales de una amplia franja de la población urbana del tipo servicios públicos, movilidad, seguridad social, entre otros.

Algunas propuestas políticas, aplicadas con ese espíritu, lo son la Biblioteca Guggenheim de Bilbao en España y el barrio Puerto Madero de Buenos Aires en Argentina. En el contexto local, se encuentra el ejemplo del dos veces intervenido parque Tercer Milenio de Bogotá D.C.

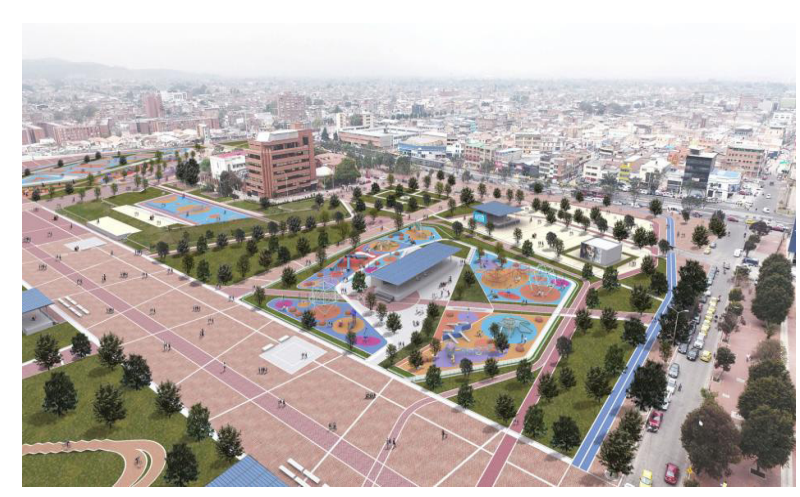

Figura 3. Proyecto de Renovación del Parque Tercer Milenio, Bogotá D.C.

Fuente: El Tiempo (2019)

\section{Reflexiones Finales}

Lo señalado hasta este punto permite identificar cómo cada uno de los paradigmas ofrece propuestas para resolver algunas de las problemáticas que fueron planteadas en el segundo apartado y que son, precisamente, las que manifiesta la Megaciudad en el marco del Cambio Climático y el reto de la Sostenibilidad.

Ahora bien, a continuación, interesa discutir en torno a aquellos aspectos que no han sido abordados, y sobre los cuales es necesario reflexionar de manera integrada. Para ello, se consideraron los tres factores explicativos de la Megaciudad que se señalaron en la introducción; estos son "Dinámicas 
de la Población y Migración", "Cambios Tecnológicos y Nuevos Estilos de Vida", "Modelos Económicos y de Ordenamiento Territorial", por considerar que al ser generales, son lo suficientemente abarcativos. Tales factores fueron subdivididos en diferentes problemáticas que se consideraron como las más urgentes de atender y que fueron explicadas en el segundo apartado ${ }^{8}$.

Una primera lectura general de la Tabla 1. nos permite ver que el paradigma de "Creación de Espacios" se muestra como la alternativa más integral de intervenir en la ciudad, en tanto que responde de manera directa o indirecta a 8 de los 10 retos planteados. En el mismo orden, la "Acupuntura Urbana" responde solamente a 4 problemas, pudiéndose considerar, entonces, como la alternativa más limitada. Además, queda manifiesta la preponderancia que tiene el factor "Modelos Económicos y de Ordenamiento Territorial", en virtud a que allí se concentran la mayoría de problemáticas de interés. Lo anterior, no justifica ignorar las otras dimensiones señaladas (Dinámicas de la Población y Migración y Cambios Tecnológicos y Nuevos Estilos de Vida), pero sí llama la atención respecto del enfoque que se requiere para la atención de los problemas de la ciudad.

Ahora bien, si se hace la lectura de la Tabla 1. desde los factores explicativos y las problemáticas señaladas, se puede concluir que la "Acupuntura Urbana" es el único paradigma que no aborda el factor demográfico en los términos de crecimiento de la población y potenciales movimientos migratorios; los otros dos si lo tienen presente dentro de los aspectos a considerar, bien sea en términos positivos o restrictivos.

Los problemas asociados a un modelo de desarrollo económico y territorial, con alta dependencia del petróleo como fuente de energía, junto con el crecimiento del parque automotor en desmedro de los sistemas de transporte público, son abordados por

8. Es importante aclarar que lo definido en este texto no agota la discusión al respecto pues el tema es más complejo de lo que aquí se puede abordar.

$\begin{array}{ll}\text { Procesos } & 12 \\ \text { Urbanos } & \end{array}$ los paradigmas de "Contención Urbana" y "Creación de Espacios", en virtud de que su perspectiva de ordenamiento territorial es más integral. Esto no ocurre con el problema de los espacios públicos de socialización, en donde el énfasis fuerte lo tienen los paradigmas de "Creación de Espacios" y "Acupuntura Urbana", los cuales incluyen dentro de sus principios la generación de espacios de calidad para la vida social de la población que habita el territorio.

Por último, respecto de la tercera dimensión, "Modelos Económicos y de Ordenamiento Territorial", se debe resaltar que los tres paradigmas responden, en diferente medida, a los retos planteados por la ocupación del suelo, así como que se adaptan a las limitaciones de las administraciones locales, en virtud a que, como se señaló en la descripción hecha más arriba, la utilización de uno u otro paradigma depende no solo de la necesidad específica sino también de las capacidades de acción de la gestión local.

El panorama cambia radicalmente al revisar el tema de preparación para desastres naturales, pues si bien en el paradigma de "Creación de Espacios" algo se trata al respecto, se puede considerar que es imposible que dé respuesta integral a los retos del calentamiento global, los cuales pueden considerarse multiescalares, pues traspasan las fronteras administrativas y de largo alcance sin una periodicidad específica.

Frente a la infraestructura instalada en la ciudad, el paradigma que mejor responde es el de "Acupuntura Urbana", desde el cual se plantea la posibilidad de renovar y reutilizar espacios deteriorados, que, de hecho, se podría considerar como la especialidad del paradigma. El tema de "Extensión de la Mancha Urbana" se presenta como el problema principal sobre el cual interviene de manera restrictiva el paradigma de "Contención Urbana", mientras que el de "Creación de Espacios" lo aborda desde un enfoque más permisivo. 
Tabla 1. Contraste de las Problemáticas de las Megaciudades, Cambio Climático y Sostenibilidad vs. Paradigmas de Intervención Analizados.

\begin{tabular}{|c|c|c|c|c|}
\hline \multirow[b]{2}{*}{ Dimensión } & \multirow[b]{2}{*}{ Problemática } & \multicolumn{3}{|c|}{ Paradigmas de Intervención Urbana } \\
\hline & & $\begin{array}{c}\text { Contención } \\
\text { Urbana }\end{array}$ & $\begin{array}{l}\text { Creación de } \\
\text { Espacios }\end{array}$ & $\begin{array}{l}\text { Acupuntura } \\
\text { Urbana }\end{array}$ \\
\hline \multirow[t]{2}{*}{$\begin{array}{c}\text { Dinámicas de la Población y } \\
\text { Migración }\end{array}$} & Crecimiento Intensivo de la Población & Si & $\mathrm{Si}$ & No \\
\hline & Dependencia del Petróleo & $\mathrm{Si}$ & $\mathrm{Si}$ & No \\
\hline \multirow{4}{*}{$\begin{array}{c}\text { Cambios Tecnológicos y Nuevos } \\
\text { Estilos de Vida }\end{array}$} & Parque Automotor & $\mathrm{Si}$ & $\mathrm{Si}$ & No \\
\hline & $\begin{array}{c}\text { Pérdida de Espacios Públicos de } \\
\text { Socialización }\end{array}$ & No & $\mathrm{Si}$ & $\mathrm{Si}$ \\
\hline & Presiones para la Ocupación de Suelos & $\mathrm{Si}$ & $\mathrm{Si}$ & $\mathrm{Si}$ \\
\hline & $\begin{array}{l}\text { Limitaciones de las Administraciones } \\
\text { Locales }\end{array}$ & Si & Si & Si \\
\hline \multirow{4}{*}{$\begin{array}{l}\text { Modelos Económicos y de } \\
\text { Ordenamiento Territorial }\end{array}$} & Deterioro de la Infraestructura Instalada & No & No & $\mathrm{Si}$ \\
\hline & $\begin{array}{l}\text { Preparación para Desastres Naturales } \\
\text { (Estrategias de mitigación-adaptación) }\end{array}$ & No & No & No \\
\hline & Extensión de la Mancha Urbana & $\mathrm{Si}$ & Si & No \\
\hline & $\begin{array}{c}\text { Contaminación de los Recursos } \\
\text { Naturales }\end{array}$ & $\mathrm{Si}$ & $\mathrm{Si}$ & No \\
\hline
\end{tabular}

Fuente: Elaboración propia

Por último, la problemática de "Contaminación de los Recursos Naturales", que no debe considerarse como exclusiva de esta dimensión, pues es transversal, tiene nuevamente respuesta desde los paradigmas de "Contención Urbana" y de "Creación de Espacios". En estos, se hace un reconocimiento explícito de la responsabilidad que tienen las ciudades en la actual crisis ambiental, por lo que se afirma que las intervenciones futuras deben ser pensadas en el marco de la Sostenibilidad del sistema urbano.

\section{¿Qué hay de una Perspectiva Integrada?}

La revisión cualitativa anterior nos permite concluir que más allá de la teoría, ningún paradigma de intervención urbana se puede considerar como suficiente para abordar las problemáticas y retos que plantea la gestión de una ciudad, y menos en el actual contexto de Megaciudades, Cambio Climático y Sustentabilidad Urbana. Así, en línea con las opiniones de otros expertos en la materia, las Megaciudades no pueden ser diseñadas ni contenidas completamente, pero sí puede guiarse su desarrollo a través de una planeación realista, aterrizada a las condiciones y necesidades específicas. Algunos expertos creen que el énfasis debe ser dar un balance adecuado entre crecimiento y estabilidad urbana, evitando los círculos viciosos del desarrollo: "equilibrar el crecimiento y la estabilidad implica cuestiones de equidad versus eficiencia, eficiencia versus empleos y equidad versus competencia global" (Jayan, 2009, p. 69) 9 .

Entidades como el Banco Asiático de Desarrollo - la ONU apuntan que los Gobiernos locales deben retornar a un crecimiento urbano planificado y bien gestionado, prestar atención a los sectores empobrecidos de las grandes ciudades, al tiempo que se trabaja el desarrollo articulado a un sistema de distribución de la actividad económica, cultural o del conocimiento de las ciudades secundarias, apostando por diversificar en núcleos más pequeños (Jayan, 2009, El País, 2008). Si bien, esta última propuesta se dificulta en países en desarrollo, en los que la gran capital sigue creciendo por ser el "único" centro de desarrollo posible, es mandatorio pensar en una alternativa más humana, sostenible y gobernable en el mediano y largo plazo.

9. "Balancing growth and stability entails questions of equity versus efficiency, efficiency versus jobs, and equity versus global competition" 
En esta línea, los Gobiernos deberían considerar las diferencias del desarrollo económico, la polarización social y calidad de la infraestructura, al tiempo que es necesario adoptar unos enfoques mínimos en políticas públicas: eficiencia, disciplina fiscal, transporte público sostenible, educación adecuada, el desarrollo de crédito y capitales, estimular la participación comunitaria en procesos de planeación urbana y basados en los desarrollos tecnológicos ecoeficientes. Aquí, la tecnología es clave para proporcionar más opciones; para la elección de mejores herramientas disponibles, para hacer frente a los retos y para la generación de nuevos mercados; $y$, de este modo, nuevas oportunidades para el desarrollo económico y el empleo (Jayan, 2009).

Revertir los efectos de fenómenos naturales, pronosticados como consecuencia del Cambio Climático, es imposible; por lo que, en este punto, las ciudades del mundo deben empezar a manejar un concepto ampliado de gestión de riesgos que incluya, no sólo la reducción de sus desechos y la producción de gases de efecto invernadero, sino que la resiliencia de las poblaciones urbanas es un concepto que debe empezar a reflejarse en todos los instrumentos de ordenación del territorio. Así, se señala la importancia de que el Cambio Climático esté integrado en las políticas del territorio, al tiempo que se debe hacer un debate serio y despolitizado frente a sus implicancias ambientales, pues, en la actualidad, son temas que se han convertido en armas políticas de los Gobiernos de turno.

Por su parte, la Sostenibilidad de las Megaciudades, $y$, en general, del sistema urbano mundial, depende de la comunicación eficaz y pertinente que se tenga con la ciudadanía, sin caer en soluciones universales ${ }^{10}$ ni mensajes catastrofistas; por el contrario, explicando con argumentos las posibilidades de actuación que hay y vinculando la responsabilidad que tenemos todos los ciudadanos, al estilo 'todos debemos ser parte de la solución'

De igual manera, es necesario que la gestión del riesgo considere la vulnerabilidad de

10. Un ejemplo de la necesidad de instaurar soluciones que propendan por la "sostenibilidad a la medida" son los indicadores adecuados de densidad urbana, pues como apunta Angel (2005), toda ciudad debe buscar una densidad que sea sustentable, aunque pueda parecer baja en comparación con otras urbes. las Megaciudades; no exclusivamente por su exposición a riesgos ambientales, sino también por la imposibilidad de hacer frente a esos impactos negativos, a través de los distintos recursos con que debería contar la ciudad. Aquí se habla, entonces, de fortalecer la administración local, la cual necesita de información actualizada frente a los efectos del Cambio Climático en su territorio; instalar las instituciones y mecanismos más adecuados para hacer diagnósticos y articular-ejecutar políticas transversales que cuenten con los instrumentos de evaluación-seguimiento necesarios, que sirvan para valorar la efectividad de las acciones desarrolladas. Algunos ejemplos de indicadores desarrollados con tal fin, son los aplicados por el Banco Interamericano de Desarrollo, para ciudades intermedias de Latinoamérica (BID, 2014).

Otros conceptos clave que deben prever la futura planeación, desarrollo y gestión urbana de amplio alcance desde la perspectiva integral de la Sostenibilidad es convertir estas ciudades en lugares ambientalmente sostenibles, inclusivas, seguras y productivas; no descuidando el factor socioeconómico en pro de una protección ambiental ideal. En este sentido, nos encontramos en un momento clave, en el que se debe valorar que la producción de ciudad, posiblemente una tendencia global imparable, necesite de paradigmas e instrumentos de intervención nuevos y creativos, que den respuesta adecuada a los principales desafíos que enfrenta la humanidad.

La protección del ambiente debe convertirse en una cuestión de supervivencia para la humanidad, pues algunos recursos naturales son limitados y no es posible crearlos artificialmente, pero, en este artículo, se hace un llamado a evaluar cómo debe hacerse dicha protección en los territorios, sin idealizar la Sostenibilidad como fin último, sino como parte del proceso de la vida de la ciudad. Para ello, la falta de recursos económicos de los Gobiernos no puede servir de excusa para no aplicar medidas correctivas y preventivas ${ }^{11}$.

\footnotetext{
11. Dos ejemplos de medidas que no generan gastos extra son: propender por consumos de energía eficientes posiblemente modificando hábitos de consumo para inducir al ahorro o utilizar la arborización urbana para el mejoramiento microclimático de zonas en riesgo y a la estructuración del ordenamiento ecológico de las ciudades.
} 
Es decir, aquí se cree que la humanidad no debe esperar hasta alcanzar una situación de crisis ambiental -consecuencia del agotamiento del suministro de agua, de la contaminación del aire, de la sedimentación del sistema de drenaje y/o de la inundación general resultante de la deforestación- para animarse a modificar comportamientos.
En este sentido, uno de los mayores retos de Sostenibilidad que enfrenta el mundo es aprender mejores maneras de tomar decisiones en conjunto sobre la dirección del cambio urbano, de manera que se promueva una mayor habitabilidad para todos los habitantes, no sólo para la minoría que pueden comprar sus propios enclaves protegidos (Sorensen y Okata, 2011; BID, 2014).

\section{REFERENCIAS}

Abba, A. (2010). Metrópolis Argentinas. Agenda política, institucionalidad y gestión de las aglomeraciones urbanas interjurisdiccionales. Buenos Aires: Editorial Café de las Ciudades.

Angel, S. (2015). Planeta de ciudades. Bogotá, Colombia: Universidad del Rosario-Lincoln Institute of Land Police.

Ainstein, L. (2012). Una aproximación sistémica a los procesos de difusión urbana: manifestaciones de la difusión, factores subyacentes, consecuencias emergentes y políticas públicas requeridas, en Ainstein, $L$ (coordinador) "Estructura urbana, institucionalidad y sustentabilidad de ciudades metropolitanas y regiones difusas. Miradas comparadas sobre Buenos Aires, Londres, Los Ángeles, París, Tokio, y Toronto". Buenos Aires: Eudeba.

Banco Interamericano para el Desarrollo - Instituto Interamericano para el Desarrollo Económico y Social (INDES). (2014). Liderando el desarrollo sostenible de las ciudades. Unidad Temática: Cambio Climático. Disponible en: https://es.slideshare.net/gracielamariani/liderando-eldesarrollo-sostenible-de-las-ciudades-unidad-temtica-cambio-climtico

Barahona, L. (2008). XS Urbano. Repensar la ciudad desde los Sistemas de Acupunturas Urbanas. Fundación de la Universidad Politécnica de Cataluña UPC.

Borsdorf, A. (2003). Cómo modelar el desarrollo y la dinámica de la ciudad latinoamericana, en EURE, 29 (086) Santiago de Chile: Pontificia Universidad Católica de Chile.

Buzai, G. (2006). Modelos urbanos. Focalización en el análisis de la estructura espacial de las ciudades de América Latina, en AAVV (2006) Crecimiento urbano y sus consecuencias sobre el entorno rural. El caso de la eco región pampeana. Buenos Aires: Orientación Gráfica Editora.

Diario El País (2008). Entrevista a Jorge Olcina. "Este nuevo siglo será el de las Megaciudadades". Disponible en: http://elpais.com/diario/2008/03/16/eps/1205652412 850215.html 
Diario El País. (2016). El 80\% de las Megaciudades del mundo está en el hemisferio sur. Disponible en: http://blogs.elpais.com/seresurbanos/2016/06/Megaciudadeshemisferiosur.html

DiarioElTiempo(2019). Losescenariosque hayenel parqueTercer Mileniotras renovación. Disponible en: https://www.eltiempo.com/bogota/parque-tercer-milenio-renovacion-2019-426344

Hansjürgens, B. y Heinrichs, D. (2014). Megacities and Climate Change: Early Adapters, Mainstream Adapters and Capacities, en Kraas, F., Aggarwal, S, Coy, M. y Mertins, G (editores) (2014). Mega cities, our global urban future. Springer.

Janoschka, M. (2002). El nuevo modelo de la ciudad latinoamericana: fragmentación y privatización, en EURE, 28 (085), 5-20. Santiago de Chile: Pontificia Universidad Católica de Chile.

Jaramillo, S. (1993). Urbanización Latinoamericana. Nuevas perspectivas. Bogotá D.C.: Ed. Escala.

Jaramillo, S. (2009). El papel del mercado del suelo en la configuración de algunos rasgos socioespaciales de las ciudades latinoamericanas, en Revista Territorios, Vol. 2 (01), 107-129. Bogotá D.C.: Universidad del Rosario.

Jayan, T. V. (2009). Megacities. Nueva Delhi, India: Vigyan Prasar.

Khanna, P. (2016). How megacities are changing the map of the world. Conferencia TED. Disponible en: https://www.ted.com/talks/parag khanna how megacities are changing the map of the world

Lerner, J. (2003). Acupuntura Urbana, Río de Janeiro-Sao Paulo, Brasil: Editora Record.

Lorrain, D. (2011). Governing megacities in emerging countries. París, Francia: Ashgate. 2011.

Mendoza, A. (2014). Gestión urbanística e institucionalidad en metrópolis de América del Sur. Munich, Alemania: Grin Verlag.

Mendoza, A. (2015). Gestión urbanística de las urbanizaciones cerradas en Colombia: el caso de Chía. Procesos Urbanos, 2(2), 58-72. https://doi.org/10.21892/2422085X.84

Organización de Naciones Unidas-Departamento de Asuntos Económicos y Sociales. (1992). Declaración de Rio sobre el Medio Ambiente y el Desarrollo. Disponible en: http://www. un.org/spanish/esa/sustdev/agenda21/riodeclaration.htm

Organización de Naciones Unidas-HABITAT. (2016). World Cities Report: Urbanization and Development. Emerging Futures. Nairobi: Kenia.

Pírez, P. (2010). La problemática de la gestión, en Atlas Ambiental de Buenos Aires.

Sáenz, H. (2006). El funcionamiento del mercado de arrendamientos en barrios populares de Bogotá. Bogotá D.C.: Lincoln Institute of Land Policy-MIMEO. 2006.

Sorensen, A. y Okata, J. (editores). (2011). Megacities: Urban form, Governance and Sustainability. Tokyo, Japón: Springer. 
Sustentabilidad Responsable. (2016). Megaciudades: el gran desafío de la Sostenibilidad. Disponible en: https://sustentabilidadresponsable.wordpress.com/2016/05/28/Megaciudades-el-grandesafio-de-la-Sostenibilidad/

Tarchópulos, D. (2006). Reseña del libro "Acupuntura Urbana", Investigaciones Geográficas, Boletín 61.

UNFPA. (2007). Estado de la población mundial 2007. Liberar el potencial del crecimiento urbano. Disponible en: https://www.unfpa.org/sites/default/files/pub-pdf/swp2007_spa.pdf 